\title{
EFEITOS DAS FASES DO CICLO MENSTRUAL E DA SÍNDROME PRÉ-MENSTRUAL SOBRE A APTIDÃO FÍSICA E PERCEPÇÃO SUB- JETIVA DE ESFORÇO EM MULHERES JOVENS
}

\author{
Victor Silveira Coswig \\ Universidade Federal do Pará, Castanhal, Pará, Brasil. \\ Jéssica Dias Da Silva \\ Universidade Federal do Pará, Castanhal, Pará, Brasil. \\ Déborah Araújo Farias \\ Universidade Federal do Pará, Castanhal, Pará, Brasil. \\ Rodolfo de Azevedo Raiol \\ Centro Universitário do Estado do Pará, Belém, Pará, Brasil. \\ Erika Cristina Murgia Estevam \\ Universidade Federal do Pará, Castanhal, Pará, Brasil.
}

\begin{abstract}
Resumo
O objetivo consistiu em investigar os efeitos das fases do Ciclo Menstrual (CM) em variáveis da aptidão física e a percepção subjetiva do esforço em mulheres jovens. Para tal, foram recrutadas 11 mulheres universitárias, que foram submetidas a testes de potência e de força dos membros superiores e inferiores e de aptidão aeróbica nas fases Folicular, Ovulatória e Lútea. Foram aplicados os testes de Arremesso de Medicine Ball, Salto Vertical e Horizontal, potência aeróbica (YoYo IRT) e de Força Máxima (1RM). Os resultados indicam que não houve diferenças significativas ( $>0,05)$ nos testes de aptidão física entre fases do CM. Concluímos que as diferentes fases do CM não influenciam o desempenho de componentes da aptidão física, seja em aspectos neuromusculares ou na potência aeróbica.
\end{abstract}

Palavras-chave: Aptidão Física. Ciclo Menstrual. Desempenho. Exercício resistido.

\section{EFFECTS OF MENSTRUAL CYCLE PHASES AND PRE-MENSTRUAL SYN- DROME IN PHYSICAL FITNESS OF YOUNG WOMEN}

\begin{abstract}
The objective was to investigate the effects of the different phases of the Menstrual Cycle $(\mathrm{CM})$ on physical fitness variables in young women. For that, 11 university women were recruited, who underwent power and strength tests for upper and lower limbs and aerobic fitness tests at Follicular, Ovulatory and Luteal phases. Medicine Ball Throw, Vertical and Horizontal Jumps, aerobic power (YoYo IRT) and maximum strength (1RM tests). The results indicate that there were no significant differences $(p>0.05)$ in physical fitness tests between any of the CM phases. We conclude that the different phases of CM do not influence the performance of physical fitness components, either in neuromuscular aspects or in aerobic power.
\end{abstract}

Keyword: Physical Fitness. Menstrual Cycle. Performance. Resistance Exercise. 


\section{EFECTOS DE LAS FASES DEL CICLO MENSTRUAL Y DEL SÍNDROME PRE- MENSTRUAL EN LA APTITUD FÍSICA Y PERCEPCIÓN SUBJETIVA DE ES- FUERZO DE MUJERES JÓVENES}

\section{Resumen}

El objetivo fue investigarlos efectos de las diferentes fases del Ciclo Menstrual (CM) en variables de aptitud física de mujeres jóvenes. Para eso, fueron reclutadas 11 mujeres universitarias, que fueron sometidas a pruebas de potencia y fuerza de los miembros superiores e inferiores y de aptitud aeróbica en las fases Folicular, de Ovulacióny Lútea. Fueron aplicadas pruebas de Lanzamiento de Medicine Ball, Salto Vertical y Horizontal, potencia aeróbica (YoYo IRT) y de Fuerza Máxima (1RM). Los resultados indican que no hubo diferencias significativas $(\mathrm{p}>0,05)$ en las pruebas de aptitud física entre las fases del CM. Concluimos que las diferentes fases del CM no influyen en el desempeño de los componentes de la aptitud física, sea en aspectos neuromusculares o en la potencia aeróbica.

Palabras Claves: Aptitud Física. Ciclo Menstrual. Desempeño. Ejercicio resistido.

\section{Introdução}

O desenvolvimento das mulheres é repleto de mudanças significativas desde a infância até a fase adulta (GALLAHUE; OZMUN; GOODWAY, 2013). Dentre essas, na adolescência ocorre a menarca, processo que inclui modificações relacionadas aos órgãos sexuais, útero, ovários, mamas, vagina e secreções de hormônios gonadotrópicos pela adenohipófise (SIMÃO et al., 2007). A partir de então, inicia-se o Ciclo Menstrual (CM), que tem em média a duração 28 dias, e é dividido em três fases: folicular, ovulatória e lútea. A fase folicular inicia a partir do primeiro dia da menstruação e dura até o nono dia; a fase ovulatória ocorre entre os dias 10 e 14; já a fase lútea inicia no fim da ovulação e dura até a nova menstruação (WOJTYS et al., 1998). A duração do CM pode variar entre mulheres ou em uma mesma mulher, visto que já foram evidenciados ciclos curtos, de apenas 20 dias, ou longos, de até 45 dias (BERNE; LEVY, 2006).

Cada fase do CM é caracterizada por secreções cíclicas de hormônios folículo estimulante (FSH) e luteinizante ( $\mathrm{LH})$ pela hipófise anterior e dos estrogênios e progesterona pelos ovários (FREITAS; MENKE, 2001). Para Guyton e Hall (1999) a fase folicular apresenta baixos níveis de estradiol e progesterona, o que ocasiona a degeneração e o desprendimento do revestimento uterino. Aproximadamente dois dias antes da ovulação, por motivos ainda não inteiramente esclarecidos; a secreção de LH e FSH pela hipófise anterior aumenta acentuadamente (GUYTON; HALL, 1999). Em seguida, inicia-se a fase lútea, onde são secretadas grandes quantidades de progesterona e estrógeno, mas principalmente progesterona (VANDER; SHERMAN; LUCIANO, 2001). No final do CM, caso o óvulo não seja fecundado, o corpo lúteo se degenera e os níveis de estrógeno e progesterona caem, provocando o início do fluxo menstrual, e um novo CM se repete (VANDER; SHERMAN; LUCIANO, 2001).

Durante o ciclo menstrual algumas mulheres podem apresentar combinações complexas de sintomas psicológicos, que incluem irritabilidade, agressão, tensão, ansiedade e depressão. Juntamente com isso acontecem mudanças somáticas como retenção de líquidos, sensibilidade mamária, dor de cabeça, sensação de inchaço e aumento de peso. Estes sintomas são decorrentes da Síndrome Pré-Menstrual (SPM) e independem de contexto social, raça ou crenças (SCHERLLENBERG, 2001). Pouco se sabe sobre os verdadeiros motivos que levam uma mulher a apresentar sintomas da SPM, no entanto, acredita-se que fatores socioeconômicos e sociais, tais como, o estilo de vida, o uso de medicamentos, tabagismo, consumo de ál- 
cool e a falta de exercício físico podem contribuir para o aparecimento dos sintomas da SPM (DEUSTER; ADERA; SOUTH-PAUL, 1999). Por outro lado, a prática de exercícios aeróbicos de intensidade moderada a vigorosa, durante seis semanas ou mais, parece reduzir sintomas da SPM e promover melhoras na aptidão aeróbica de mulheres no período menstrual (VISHNUPRIVA; RAJARAJESWARAM, 2011).

Neste sentido, mulheres que praticam exercício físico tendem a diminuir os sintomas da SPM, o que se deve principalmente às melhoras na circulação sanguínea (TIMONEN et al., 1971). De outro modo, podem ocorrer variações em alguns componentes da aptidão física nas diferentes fases do CM (LEITÃO et al., 2000). Quanto a força muscular, Pallavi, Souza e Shivaprakash (2017) afirmam que as mulheres apresentam maior volume de trabalho muscular realizado e menor taxa de fadiga durante a fase folicular e as mudanças ocorridas no período menstrual podem afetar o desempenho no exercício físico. Em contrapartida, Simão et al. (2007) verificaram que na fase folicular as mulheres apresentam redução significativa de força, o que pôde ser apurado a partir do teste de $8 \mathrm{RM}$ para membros inferiores que foi realizado no leg press. Quando considerado o consumo máximo de oxigênio $\left(\mathrm{VO}_{2} \mathrm{max}\right)$, Lamina; Hanif e Muhammed (2011) sugerem que as fases do CM não afetam o desempenho aeróbio curto de intensidade máxima.

Neste contexto, faz-se necessário investigar a relação entre aptidão física e as diferentes fases do CM, haja vista que os estudos supracitados apresentam resultados conflitantes e, ao conhecimento dos autores, não foram identificadas pesquisas que avaliassem elementos da aptidão física e CM de forma simultânea. Diante disso, o objetivo do presente estudo consistiu em investigar os efeitos das diferentes fases do CM e dos sintomas da SPM sobre a força de membros superiores (MMSS) e inferiores (MMII), potência aeróbica e percepção subjetiva de esforço em mulheres jovens ativas.

\section{Materiais e métodos}

\section{Tipo de pesquisa e variáveis}

A presente pesquisa caracteriza-se como observacional transversal analítica (THOMAS; NELSON; SILVERMAN, 2002) e foi aprovada pelo comitê de ética local (037/2011). Foram consideradas variáveis dependentes, aquelas que são relacionadas à aptidão física (força e potência de membros inferiores e superiores e a potência aeróbia), e independentes às fases do CM (Lútea, Folicular e Ovulatória).

\section{Descrição da Amostra}

De acordo com o cálculo amostral realizado por meio do software G*Power versão 3.0.10, e a partir de dados prévios com salto horizontal (COSWIG; RAMOS; DEL VECCHI$\mathrm{O}, 2016$ ), para tamanho de efeito de $\mathrm{F}$ de 0,50 , alfa menor que 0,05 e poder de 0.90 , seriam necessários 11 indivíduos. A amostra foi composta por mulheres com idade entre 18 e 36 anos, praticantes de treinamento resistido a, ao menos, três meses ininterruptos, com ciclos menstruais regulares de 26 a 30 dias, que apresentavam sintomas decorrentes da SPM e que faziam uso de contraceptivos orais, sendo este último critério aderido pelo fato de que a utilização deste medicamento auxilia na regulação do ciclo menstrual, permitindo uma facilitação de identificação do início de cada fase do ciclo. Foram excluídas aquelas que, por qualquer motivo, não completaram a bateria de testes físicos ou declararam uso de substâncias que pudessem afetar os resultados, tais como consumo de álcool, bebidas energéticas, ergogênicos farmacológicos ou outros. 


\section{Delineamento}

O contato com as voluntárias ocorreu por conveniência, através de convite verbal e considerando a disponibilidade pessoal e material, mediante a assinatura do termo de consentimento livre e esclarecido (TCLE). Foi realizada uma bateria de testes entre o primeiro e terceiro dia de cada fase do CM de modo aleatório, totalizando 3 baterias de testes ao longo do estudo. A primeira bateria foi executada na fase folicular, período que compreende do início da menstruação até o nono dia; a seguinte, na fase ovulatória, a qual corresponde ao período do décimo ao décimo quarto dia; e então, na fase lútea, cuja duração perdura do décimo quinto dia até o início da próxima menstruação. Inicialmente foram apresentados às voluntárias os objetivos, os riscos e os benefícios decorrentes da participação no estudo; elas assinaram o TCLE e foi aplicado o questionário de Rastreamento de Síndrome Pré-Menstrual (PSST); elas responderam à anamnese contendo dados demográficos (nome, idade e tempo de prática de treinamento de força), e em seguida iniciaram as medidas antropométricas e, posteriormente, os testes físicos (Arremesso de Medicine Ball; Salto Horizontal; Salto Vertical; Yo-Yo Intermitente Recovery Test e de uma Repetição máxima - 1RM) com o intervalo de três minutos entre eles. No segundo e no terceiro encontro as voluntárias repetiram as baterias de testes.

\section{Procedimentos}

Rastreamento de Síndrome Pré-Menstrual (PSST): uma escala de autoaplicação recordatória, com 19 itens (14 referentes aos sintomas e cinco questões relacionadas às consequências dos sintomas) que medem a gravidade dos sintomas da SPM em graduações de: "Nada", "Leve", "Moderado" e "Severo"; cada item recebe uma resposta única assinalada ao lado, sendo preenchidos em um único momento na pesquisa, durante a triagem. Para o diagnóstico de SPM a paciente deve apresentar ao menos um sintoma nos itens de 1-4, moderado a severo; pelo menos quatro sintomas nos itens de 1-14, moderado a severo; e ao menos um item de A-E precisa ser moderado a severo para ter o diagnóstico de SPM (HENZ, 2016).

Avaliações Antropométricas: Para mensurar a massa corporal das voluntárias recorreuse a uma balança com plataforma (GTech), com a precisão de $0,1 \mathrm{~kg}$. Já para medir a estatura utilizou-se uma trena métrica com precisão de $2 \mathrm{~mm}$, de acordo com os procedimentos descritos por Gaia et al. (2012).

Potência de membros superiores (Arremesso de Medicine Ball): Utilizando uma Medicine Ball de $3 \mathrm{~kg}$ e uma trena de 10 metros fixada no solo perpendicularmente à parede, a voluntária sentou-se com os joelhos estendidos, as pernas unidas e as costas completamente apoiadas à parede, segurando a Medicine Ball junto ao peito com os cotovelos flexionados. Ao sinal do avaliador, a voluntária lançou a bola à maior distância possível, movimentando apenas seus braços. A distância do arremesso foi registrada a partir do ponto zero até o local em que a bola tocou o solo pela primeira vez. Após a realização de três tentativas com o intervalo de 30 segundos foi considerado o maior valor para análise (MACKENZIE, 2005).

Potência de membros inferiores (Impulsão Horizontal): Utilizou-se uma fita métrica, não elástica, previamente fixada no solo, perpendicular à linha de salto. A voluntária com os pés separados e paralelos, distantes 20 centímetros um do outro, posicionou-se atrás da linha de saída demarcada no solo. Na preparação para o salto a voluntária realizou flexão de ombros, posicionou os braços para trás do tronco e flexionou os joelhos. O salto foi efetivado com a voluntária estendendo os membros inferiores durante o movimento, estendendo a articulação dos ombros e projetando o tronco para frente. A medida foi realizada da linha de saída até onde a primeira parte do corpo da voluntária, os dedos dos pés, tocou o solo. Após a 
realização de três tentativas com o intervalo de 30 segundos, o maior valor obtido foi utilizado nas análises (MACKENZIE, 2005).

Potência de membros inferiores (Salto Vertical - Sargent Jump Test): com a voluntária em pé e com os pés juntos, foi posto giz nas pontas dos dedos da mão dominante; a voluntária elevou o braço e fez uma marca no ponto mais alto possível conservando os calcanhares em contato com o solo. A voluntária fez uma marca na parede com os dedos sujos (sujos de giz), agachou-se e saltou, fazendo nova marca com os dedos na parede no ponto mais alto que se conseguiu alcançar. Não era permitido andar ou tomar distância para saltar. O resultado foi registrado medindo-se a distância entre a primeira marca e a segunda, após a realização de três tentativas com o intervalo de 30 segundos, o maior valor foi considerado para análise (MACKENZIE, 2005).

Potência aeróbia (Yo-Yo Intermittent Recovery Test- Level 1): o teste foi realizado em uma quadra poliesportiva. Cones foram utilizados para demarcar o espaço para corrida. As voluntárias deveriam percorrer a distância de $20 \mathrm{~m}$, ida e volta $(40 \mathrm{~m})$, dentro do tempo estipulado por um sinal sonoro. O intervalo de tempo foi diminuindo ao longo do teste, aumentando a intensidade da corrida. As participantes tiveram 10 segundos de intervalo entre cada esforço, e neste tempo deveriam trotar até o cone do recuo e se posicionar para uma nova saída. O teste foi interrompido quando as voluntárias não conseguiram mais suportar a intensidade de corrida (LIZANA et al., 2014).

Teste de uma Repetição Máxima (1RM): Para a determinação da força muscular foram realizados testes de 1RM no Supino Reto e no Leg Press. O teste do Supino iniciou com a voluntária deitada em decúbito dorsal sobre o banco com os joelhos flexionados e pés apoiados. O teste no Leg Press iniciou-se na posição sentada com os pés apoiados na plataforma. A voluntária realizou um aquecimento de oito repetições a 50\% de 1RM percebida, seguido de três repetições a 70\% de 1RM percebida. Após $5 \mathrm{~min}$. de intervalo, realizou-se o teste de 1RM, acrescentando-se, quando necessário, de 0,4 a $5 \mathrm{~kg}$, totalizando de três a cinco tentativas com o intervalo de 3 a 5 minutos entre eles. Registrou-se como carga máxima aquela levantada em um único movimento. Os testes foram conduzidos conforme o protocolo proposto por Materko, Neves e Santos (2007).

\section{Análise estatística}

Após a verificação da normalidade dos dados através do teste de Shapiro-Wilk, a média é utilizada como medida de centralidade e o desvio padrão como medida de dispersão. Para comparações entre as médias foi aplicado o teste ANOVA de uma via para medidas repetidas com post-hoc de Bonferroni. Adicionalmente foram calculados os tamanhos de efeito (TE) por meio do $d$ de Cohen e classificados como trivial $(<0,2)$, pequeno $(0,2$ a 0,3$)$, médio $(0,4$ a 0,7$)$ e grande $(>0,8)$. A significância estatística foi determinada quando $p<0,05$ e as análises foram executadas no software SPSS versão 22.0.

\section{Resultados}

A amostra final analisada foi composta por 11 mulheres com idade média de 27,5 \pm 4,6 anos, massa corporal de 54,7 $\pm 6 \mathrm{~kg}$ e estatura de 1,57 $\pm 0,07 \mathrm{~cm}$. Na Tabela 1 são apresentados os dados descritivos de acordo com diagnóstico da SPM, na qual se evidencia a presença de SPM em todas as participantes, com maiores fatores de efeito severo em sintomas físi$\cos (72,7 \%)$, seguidos de falta de interesse em atividades sociais e raiva/irritabilidade (45,5\%). Já o desempenho nos testes físicos é apresentado na Tabela 2, na qual se indica a ausência de diferença entre os momentos de análise de acordo com as fases do CM. Para todas as situações os tamanhos de efeito entre as fases foram classificados como triviais ou pe- 
quenos $(<0,3)$, com exceção da PSE que apresentou efeito médio entre as fases folicular e ovulatória $(\mathrm{TE}=0,4)$ e efeito grande entre as fases ovulatória e lútea $(\mathrm{TE}=0,6)$ e lútea e folicular $(\mathrm{TE}=0,6)$. Ainda, os deltas percentuais de variação do desempenho em testes físicos, entre fases do CM, estão apresentados na Figura 1. Nela, percebe-se a ausência de diferença em todas as situações.

Tabela 1 - Prevalência de respostas ao PSST de acordo com a severidade dos sintomas (n=11)

\begin{tabular}{lcccccccc}
\hline & \multicolumn{3}{c}{ Nada } & \multicolumn{3}{c}{ Leve } & \multicolumn{3}{c}{ Moderado } & \multicolumn{2}{c}{ Severo } \\
& $\mathrm{n}$ & $\%$ & $\mathrm{n}$ & $\%$ & $\mathrm{n}$ & $\%$ & $\mathrm{n}$ & $\%$ \\
\hline Raiva/Irritabilidade & 1 & 9,1 & 2 & 18,2 & 3 & 27,3 & 5 & 45,5 \\
Ansiedade/Tensão & 1 & 9,1 & 2 & 18,2 & 5 & 45,5 & 3 & 27,3 \\
Chorosa/ Mais sensível a rejeição & 2 & 18,2 & 2 & 18,2 & 5 & 45,5 & 2 & 18,2 \\
Humor depressivo e sem esperança & 2 & 18,2 & 7 & 63,6 & 0 & 0,0 & 0 & 0,0 \\
Falta de interesse em atividades no trabalho & 0 & 0,0 & 5 & 45,5 & 6 & 54,5 & 0 & 0,0 \\
Falta de interesse em atividades de casa & 0 & 0,0 & 1 & 9,1 & 9 & 81,8 & 1 & 9,1 \\
Falta de interesse em atividades sociais & 0 & 0,0 & 2 & 18,2 & 4 & 36,4 & 5 & 45,5 \\
Dificuldade de concentração & 0 & 0,0 & 4 & 36,4 & 7 & 63,6 & 0 & 0,0 \\
Fadiga/ Falta de energia & 0 & 0,0 & 2 & 18,2 & 7 & 63,6 & 2 & 18,2 \\
Comendo demais/ Desejo de comer & 0 & 0,0 & 4 & 36,4 & 4 & 36,4 & 2 & 18,2 \\
Insônia & 1 & 9,1 & 7 & 63,6 & 2 & 18,2 & 1 & 9,1 \\
Hipersônia & 2 & 18,2 & 1 & 9,1 & 5 & 45,5 & 3 & 27,3 \\
Sentindo-se sobre pressão ou fora de con- & & & & & & & & \\
trole & 6 & 54,5 & 4 & 36,4 & 1 & 9,1 & 0 & 0,0 \\
Sintomas físicos & 0 & 0,0 & 0 & 0,0 & 3 & 27,3 & 8 & 72,7 \\
Seus sintomas acima interferem com: & & & & & & & & \\
Sua eficiência de produtividade no trabalho & 0 & 0,0 & 4 & 36,3 & 7 & 63,6 & 0 & 0,0 \\
Seus relacionamentos com colegas no tra- & & & & & & & & \\
balho & 0 & 0,0 & 8 & 72,7 & 3 & 27,2 & 0 & 0,0 \\
Seus relacionamentos familiares & 0 & 0,0 & 8 & 72,7 & 3 & 27,2 & 0 & 0,0 \\
Suas atividades e vida social & 0 & 0,0 & 0 & 0,0 & 6 & 54,5 & 5 & 45,4 \\
Suas responsabilidades em casa & 0 & 0,0 & 0 & 0,0 & 8 & 72,7 & 3 & 27,2 \\
\hline & Fonte: Elaborada pelo autor, 2018. & & & &
\end{tabular}

Tabela 2 - Desempenho físico de acordo com as fases do ciclo menstrual ( $\mathrm{n}=11)$ Média \pm dp

\begin{tabular}{|c|c|c|c|c|c|c|}
\hline & Folicular & Ovulatória & Lútea & $\mathrm{F}$ & $\eta^{2}$ & $\mathrm{P}$ \\
\hline $\mathrm{AMB}(\mathrm{m})$ & $2,8 \pm 0,4$ & $2,8 \pm 0,4$ & $2,9 \pm 0,4$ & 1,04 & 0,09 & 0,37 \\
\hline $\begin{array}{l}\text { Salto Horizontal } \\
\text { (m) }\end{array}$ & $1,7 \pm 0,4$ & $1,7 \pm 0,4$ & $1,6 \pm 0,2$ & 0,44 & 0,04 & 0,64 \\
\hline Salto Vertical $(\mathrm{cm})$ & $0,4 \pm 0,1$ & $0,3 \pm 0,1$ & $0,3 \pm 0,1$ & 2,22 & 0,18 & 0,13 \\
\hline Yo-Yo IRT1 (m) & $37,1 \pm 2,4$ & $36,9 \pm 2,3$ & $36,8 \pm 2,3$ & 2,52 & 0,20 & 0,10 \\
\hline 1RM Supino (kg) & $29,3 \pm 6,7$ & $31,0 \pm 6,7$ & $29,0 \pm 8,1$ & 0,40 & 0,12 & 0,26 \\
\hline $\begin{array}{l}\text { 1RM Leg Press } \\
(\mathrm{kg})\end{array}$ & $175,5 \pm 43,0$ & $170,5 \pm 44,5$ & $171,8 \pm 47,3$ & 0,81 & 0,07 & 0,45 \\
\hline PSE (u.a.) & $7,5 \pm 0,8$ & $7,1 \pm 0,3$ & $6,9 \pm 0,3$ & 2,69 & 0,21 & 0,09 \\
\hline
\end{tabular}

AMB: Arremesso de Medicine Ball; PSE: Percepção Subjetiva de Esforço; RM: Repetições Máximas; IRT1: Intermittent Recovery Test Level 1. 
Fonte: Elaborada pelo autor, 2018.

Figura 1 - Deltas de variação entre fases do CM de acordo com os testes físicos $(n=11)$

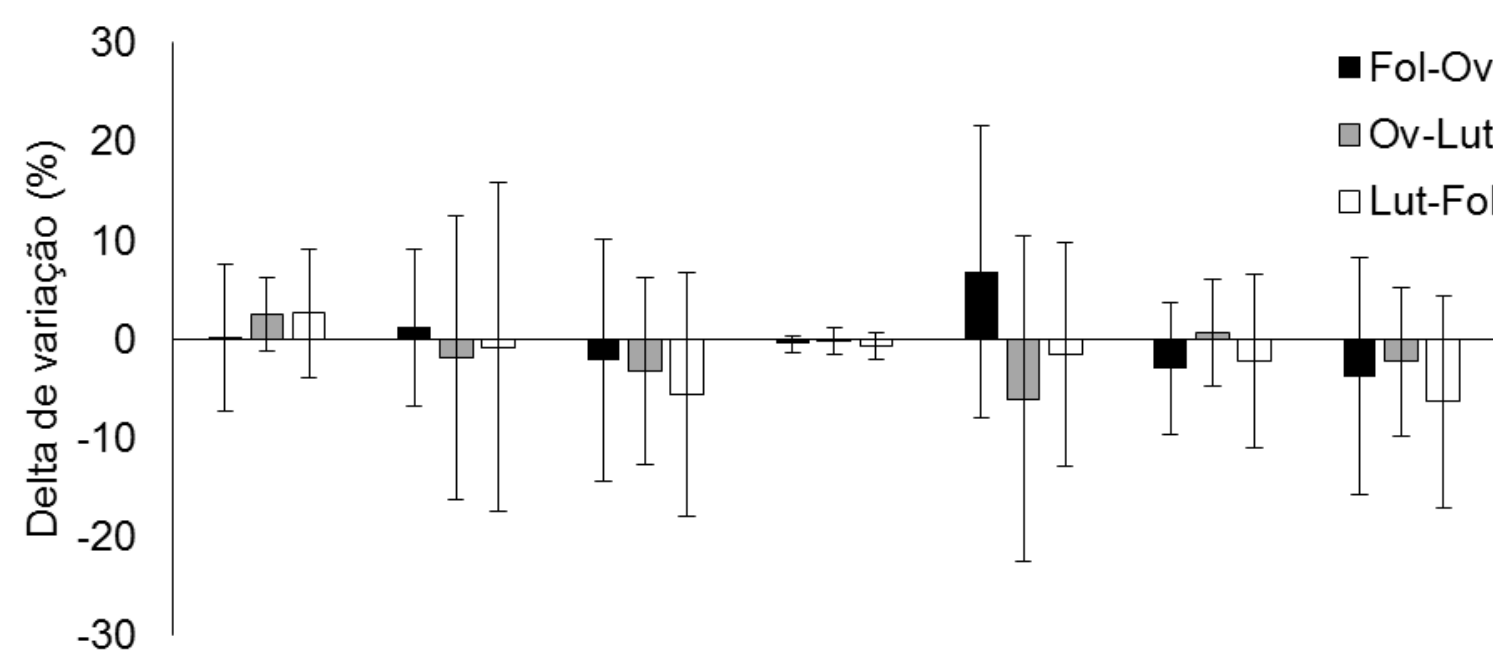

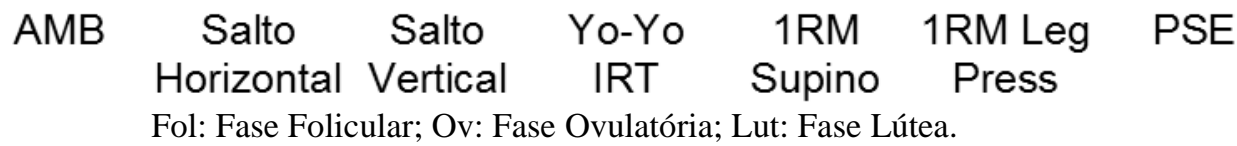

Fonte: Elaborada pelo autor, 2018.

\section{Discussão}

O presente estudo objetivou investigar o comportamento de componentes da aptidão física durante as diferentes fases do CM. Para realizar a análise dos dados obtidos com a investigação consideraram-se mulheres adultas, praticantes de treinamento resistido, com ciclos menstruais regulares, que apresentavam sintomas recorrentes da SPM e que faziam uso de contraceptivos orais. A partir dos resultados, os principais achados indicam que não há influência das fases do CM sobre a força, potência de membros superiores e inferiores e potência aeróbia de mulheres jovens treinadas.

Após aplicação do PSST constatamos que todas as 11 voluntárias apresentaram sintomas severos da SPM e que os maiores fatores desse diagnóstico estavam relacionados com sintomas físicos $(72,7 \%)$, seguidos de falta de interesse em atividades sociais e raiva/irritabilidade (45,5\%). Segundo Bathia e Bhatia (2002), esses sintomas são recorrentes principalmente durante a fase lútea do ciclo menstrual e interferem de maneira expressiva em aspectos sociais, ocupacionais e sexuais. Estudos prévios em mulheres treinadas e destreinadas analisaram os impactos do CM sobre o desempenho de exercícios físicos e não encontraram alterações significativas em componentes da aptidão física, tais como força muscular, flexibilidade, potência aeróbia e potência anaeróbia (CHAVES; SIMÃO; ARÁUJO, 2002; MELEGÁRIO et al., 2006; CONSTANTINI; DUBNOV-RAZ; LEBRUN, 2005; JONGE, 2003).

Os sintomas pré-menstruais ou dismenorreia parecem exercer efeito negativo no desempenho de mulheres, podendo afetar a força muscular devido às flutuações nos níveis dos hormônios sexuais progesterona e estrógeno (GIACOMONI et al., 2000; SIMÃO et al., 
2007). Os achados do presente estudo não verificaram diferenças significativas nas fases do CM, em componentes da aptidão física. Por outro lado, Gaion, Vieira e Da Silva (2008), após estudarem 112 atletas adultas que não faziam uso de contraceptivo oral, constataram que a prevalência de SPM apresenta impacto negativo no desempenho esportivo percebido de atletas brasileiras de futsal, tendo as atletas, relatado cansaço, irritabilidade, depressão, dores lombares, mastalgia e dificuldades de concentração. Porém, é importante ressaltar que as participantes do presente estudo não eram atletas e faziam uso de contraceptivo oral, o que pode ser um fator responsável para o contraste de resultados com o estudo supracitado, especialmente devido ao estresse psicológico competitivo a que as atletas são expostas.

$\mathrm{Na}$ fase lútea observa-se um aumento das concentrações de progesterona, o que sugere um desempenho negativo durante a prática do exercício, já durante a fase ovulatória, os níveis elevados de estrogênio e a secreção de noradrenalina são maiores, o que resultaria em um aumento no desempenho (GUYTON; HALL, 1999). De acordo com Pallavi, Souza e Shivaprakash (2017), na fase folicular há maior produção de trabalho e força e menor taxa de fadiga em comparação com as fases ovulatória e lútea. O que foi reforçado por Jhonson (2008) e Lamina, Hanif e Muhammed (2011) que afirmaram que há um melhor desempenho de componentes da aptidão física devido às variações cíclicas hormonais reprodutivas endógenas que aumentam a força e a resistência muscular na fase folicular do CM. Porém, no presente estudo, não foram observadas modificações expressivas em nenhum dos testes realizados com as voluntárias durante as diferentes fases do CM, dessa forma, não havendo queda de desempenho. Corroborando com o presente estudo, Sakamaki-Sunaga et al. (2016) analisaram os efeitos de diferentes frequências de treinamento resistido de alta intensidade sobre a força de MMSS em diferentes fases do ciclo menstrual. As variáveis analisadas foram a área de secção transversa, força máxima e contração voluntária máxima durante as fases folicular e lútea, não sendo observadas diferenças significativas entre as fases. Vale ainda ressaltar que o presente estudo utilizou participantes que faziam uso de contraceptivos orais, ao contrário do estudo supracitado, porém ambos não apresentaram diferenças significativas para a variável força nas diferentes fases do CM, o que indica que a utilização de contraceptivos orais não parece influenciar negativamente o desempenho de força independente da fase do CM.

Dessa forma, quanto à força muscular, não foi possível observar alterações expressivas em nenhumas das fases do CM no presente estudo. Dias, Simão e Silva Novaes (2005), através de testes de 10RM, avaliaram a força muscular de oito jovens usuárias de contraceptivos orais e que praticavam treinamento de força por ao menos 6 meses consecutivos. Após a aplicação dos testes que foram realizados entre o primeiro e terceiro dia de cada fase do CM, não foram observadas variações significativas na força muscular máxima durante as distintas fases do CM, o que corrobora com nosso estudo.

Por outro lado, Loureiro et al. (2011), ao analisar a força de nove mulheres adultas através do teste de 10RM no leg press $45^{\circ}$, supino horizontal, cadeira extensora e rosca bíceps, constataram que no exercício leg press $45^{\circ}$ houve incremento de $5 \%$ de força muscular na fase lútea quando comparado às outras fases. Os autores indicam que isso pode ter ocorrido por adaptações ao teste ao longo das coletas; ao nível de motivação entre avaliada e avaliador; aos aumentos nos níveis de força e à quantidade da massa muscular envolvida na execução do movimento. É importante ressaltar que no estudo supracitado, os testes foram realizados entre o primeiro e quinto dia de cada fase do CM. Os mesmos resultados mostrando superioridade da fase lútea em relação às demais foram encontrados por Sakamaki, Yasuda e Abe (2012) ao analisarem oito mulheres eumenorréicas. Wikström-Frisén et al. (2017a) constataram que as duas primeiras semanas do CM podem induzir efeitos benéficos maiores para aplicação de exercícios de alta intensidade pois proporcionam aumento da força, potência e aumento da massa magra em MMII. Por outro lado, o mesmo grupo de pesquisadores avaliou os potenciais efeitos negativos de um treinamento periodizado de alta intensidade para MMII em mulhe- 
res usuárias de contraceptivo oral durante o $\mathrm{CM}$ sobre a tríade da mulher atleta (WIKSTRÖM-FRISÉN; BORAXBEKK; HENRIKSSON-LARSÉN, 2017b). Os autores concluíram que o treinamento não foi associado a consequências negativas relacionadas ao exercício em componentes da tríade da mulher atleta e que não há a necessidade de ajustar o treinamento para cada fase do CM no intuito de maximizar o desempenho, o que concorda com os achados aqui apresentados.

Em relação a potência aeróbia, assim como o presente estudo, outros trabalhos mostram que o CM não interfere na aptidão aeróbia, pois as concentrações séricas de estrogênio/progesterona não seriam suficientes para afetar o desempenho físico e causar alterações determinantes no consumo máximo de oxigênio (VO2máx) (FRIDÉN; HIRSCHBERG; SAARTOK, 2003; LEITÃO et al., 2000; SARWAR; NICLOS; RUTHERFORD, 1996; JONGE, 2003). De Souza et al. (1990) estudaram oito corredoras que realizaram corrida em esteira (40min a $80 \%$ de VO2máx) durante as fases folicular e lútea (6-8 d de LH), e concluíram que nem a fase menstrual (folicular vs lútea) nem o estado menstrual (eumenorréica vs amenorréia) alteram ou limitam o desempenho do exercício em atletas do sexo feminino, pois o CM não afeta as características contráteis dos músculos e também não causa alterações expressivas na aptidão aeróbia (JONGE, 2003; LAMINA; HANIF; MUHAMMED, 2011). Em contrapartida, a utilização de contraceptivo oral pode reduzir o VO2máx (LEBRUN et al., 2003). Schaumberg et al. (2017) examinaram a influência do uso de contraceptivo oral sobre o pico de potência, VO2máx e pico de débito cardíaco após treinamento de Sprint intervalado (SIT) em mulheres recreacionalmente ativas. Participaram do estudo 41 mulheres, sendo divididas em grupo contraceptivo oral $(n=25)$ e grupo controle $(n=16)$. Os autores constataram que o uso de contraceptivos orais resultou em uma redução do VO2máx e pico de débito cardíaco, concluindo assim que a utilização de contraceptivos orais pode influenciar no período de adaptação fisiológica. Vale ressaltar que diferentes metodologias foram utilizadas nos estudos supracitados, o que torna difícil a comparação entre resultados.

Considerando a PSE, não foram observadas alterações significativas em nenhuma das fases do CM. Apesar disso, cabe destacar que, se considerados os cálculos de TE, percebe-se redução da PSE na fase lútea em comparação às demais, com efeito médio. Esta resposta poderia ser justificada pela maior taxa de fadiga apresentada na fase lútea (PALLAVI; SOUZA; SHIVAPRAKASH, 2017), o que reduziria a produção de trabalho e, consequentemente, a PSE. Em contrapartida, Fortes et al. (2015), após estudarem a influência do CM na força muscular e na PSE em atletas de natação usuárias de contraceptivos orais, constataram que a PSE foi maior na fase lútea em relação a fase folicular, sendo que não foi observada variação na carga de 10RM. Segundo os autores, esses resultados mostram que as variações na PSE e na força muscular durante as diferentes fases do CM podem não apresentar o mesmo padrão, o que diverge de nossos achados.

Algumas limitações devem ser consideradas: Após o estabelecimento dos critérios de inclusão na pesquisa, 22 mulheres se voluntariaram para participar do estudo. Entretanto, à medida que as coletas foram conduzidas, ocorreu uma perda amostral de $50 \%$ e o experimento foi finalizado com onze mulheres. Apesar da perda amostral expressiva, este fato não invalida nossos achados, visto que o número de pessoas atende ao número mínimo indicado pelo cálculo amostral.

O tempo de coleta também deve ser considerado, uma vez que os dados foram coletados em um único CM. Dessa forma, sugere-se a estudos futuros que reproduzam este protocolo em CM subsequentes. Outra limitação refere-se à definição do início e do final de cada fase do CM, visto que a maior precisão na determinação do ciclo por medidas dos níveis séricos da razão estrogênio/progesterona na urina ou no sangue poderia ser sugerida. Por outro lado, o presente trabalho segue protocolos previamente aplicados e, conforme anteriormente mencionado, acredita-se que as dosagens hormonais não tenham efeito determinante nas vari- 
ações de desempenho físico (FRIDÉN; HIRSCHBERG; SAARTOK, 2003; LEITÃO et al., 2000; SARWAR; NICLOS; RUTHERFORD, 1996; JONGE, 2003).

Destaca-se que as evidências são controversas quanto aos efeitos das diferentes fases do CM sobre o desempenho de componentes da aptidão física, entretanto, existem poucos estudos sobre os efeitos do CM em componentes da aptidão física de forma simultânea. Portanto, sugere-se a realização de novos estudos que apresentem métodos similares, mas com o número de sujeitos maior, além de explorar a interação entre as distintas fases do CM para que assim seja possível uma comparação e análise mais detalhada dessa lacuna evidenciada. De toda forma, o presente trabalho pode servir para auxiliar treinadores, especialmente em nível amador, dada a característica da amostra, a prescreverem e periodizarem os treinamentos de suas atletas.

\section{Conclusão}

As diferentes fases do CM não influenciam o desempenho de componentes da aptidão física, seja em aspectos neuromusculares ou na potência aeróbica. Esses achados podem auxiliar treinadores na prescrição do treinamento de suas equipes ou atletas, pois mesmo que ocorra alguma mudança no estado psicológico ou humor, o desempenho físico não tende a ser afetado. Por outro lado, os TE apresentados das variações de PSE reforçam a indicação de monitoramento constante desta variável. Assim, parece não haver necessidade de modificação do treinamento ou de datas de avaliações de acordo com a fase do CM.

\section{Referências}

ACIOLY, P. L. Estilo de vida e insatisfação referida quanto ao trabalho entre BERNE, R. M.; LEVY, M. N. Fundamentos de Fisiologia. Rio de Janeiro: Elsevier, 2006.

BHATIA, S. C.; BHATIA, S. K. Diagnosis and treatment of premenstrual dysphoric disorder. American Family Physician, v. 66, n.7, p. 1239-1248, 2002.

CHAVES, C. P. G.; SIMÃO, R.; ARAÚJO, C. G. S. de. Ausência de variação da flexibilidade durante o ciclo menstrual em universitárias. Revista Brasileira de Medicina do Esporte, v. 8, n. 6, p. 212-8, 2002.

CONSTANTINI, N. W.; DUBNOV-RAZ, G.; LEBRUN, C. M. The menstrual cycle and sport performance. Clinical Sports Medicine, v. 24, n. 2, p. 51-82, 2005.

COSWIG, V. S.; RAMOS, S. de P.; DEL VECCHIO, F. B. Time-motion and biological responses in simulated mixed martial arts sparring matches. Journal of Strength and Conditioning Research, v. 30, n. 8, p. 2156-2163, 2016.

DE SOUZA, M. J. et al. Effects of menstrual phase and amenorrhea on exercise performance in runners. Medicine and science in sports and exercise, v. 22, n. 5, p. 575-580, 1990.

DEUSTER, P. A.; ADERA, T.; SOUTH-PAUL, J. Biological, social, and behavioral factors associated with premenstrual syndrome. Archives of family medicine, v. 8, n. 2, p. 122 , 1999. 
DIAS, I.; SIMÃO, R.; SILVA NOVAES, J. da. Efeito das Diferentes Fases do Ciclo Menstrual em um Teste de 10RM. Fitness \& Performance Journal, v. 4, n. 5, p. 288-292, 2005.

FLECK, S. J.; KRAEMER, W. J. Fundamentos do treinamento de força muscular. Porto Alegre: Artmed, 2006.

FORTES, L. S. et al. Influência do ciclo menstrual na força muscular e percepção subjetiva do esforço em atletas de natação que utilizam contraceptivos-DOI. Revista Brasileira de Ciência e Movimento, v. 23, n. 3, p. 81-87, 2015.

FREITAS, F.; MENKE, C. H. Rotinas em Ginecologia. Porto Alegre: Art med, 2001.

FRIDÉN, C.; HIRSCHBERG, A. L.; SAARTOK, T. Muscle strength and endurance do not significantly vary across 3 phases of the menstrual cycle in moderately active premenopausal women. Clinical Journal of Sport Medicine, v. 13, n. 4, p. 238-241, 2003.

GAION, P. A.; VIEIRA, L. F.; DA SILVA, C. M. L. Premenstrual syndrome and perception of impact on sport performance from brazilian indoor soccer athletes. Brazilian Journal of Kinanthropometry and Human Performance, v. 11, n. 1, p. 73-80, 2008.

GALlAHUE, D. L.; OZMUN, J. C.; GOODWAY, J. D. Compreendendo o desenvolvimento motor: bebês, crianças, adolescentes e adultos. 7 ed. Porto Alegre: AMGH, 2013.

GAYA, A. et al. Projeto Esporte Brasil: PROESP-Br: manual de testes e avaliação: versão 2012. Porto Alegre: UFRGS/CNPq/Ministério do Esporte, 2012.

GIACOMONI, M. et al. Influence of the menstrual cycle phase and menstrual symptoms on maximal anaerobic performance. Medicine \& Science in Sports \& Exercise, v. 32, n. 2, p. 486, 2000.

GUYTON, A. C.; HALL, J. E. Fisiologia humana e mecanismo das doenças. Rio de Janeiro: Guanabara Koogan, 1999.

HENZ, A. Diagnóstico da síndrome pré-menstrual: comparação de dois instrumentosregistro diário da intensidade dos problemas (DRSP) e instrumento de rastreamento de sintomas pré-menstruais (PSST). 2016. 81f. Dissertação (Mestrado em Ciências da Saúde) - Programa de Pós-Graduação em Ciências da Saúde: Ginecologia e Obstetrícia, Faculdade de Medicina, Universidade Federal do Rio Grande do Sul, Porto Alegre, 2016.

JOHNSON, T. Knowledge and Attitudes Regarding the Menstrual Cycle, Oral Contraceptives, and Sport Performance: The Conceptualization and Development of a Questionnaire for Athletic Coaches. Dissertation (Doctor of Philosophy). Department of Educational Psychology and Learning Systems. Florida State University; 2008.

JONGE, A. K. X. J. de. Effects of the menstrual cycle on exercise performance. Sports Medicine, v. 33, n. 11, p. 833-851, 2003.

LAMINA, S.; HANIF, S.; MUHAMMED, H. Influence of menstrual cycle on maximal aerobic power of young female adults. African Journal of Physiotherapy and Rehabilitation Sciences, v. 3, n. 1, p. 36-41, 2011. 
LEBRUN, C. M. et al . Decreased maximal aerobic capacity with use of a triphasic oral contraceptive in highly active women: a randomised controlled trial. British Journal of Sports Medicine, v. 37, n. 4, p. 315-20, 2003.

LEITÃO, M. B. et al. Posicionamento oficial da Sociedade Brasileira de Medicina do Esporte: atividade física e saúde na mulher. Revista Brasileira de Medicina do Esporte, v. 6, n. 6, p. $215-220,2000$.

LIZANA, C. J. R. et al. Análise da potência aeróbia de futebolistas por meio de teste de campo e teste laboratorial. Revista Brasileira de Medicina do Esporte, v. 20, n. 6, p. 447-450, 2014.

LOUREIRO, S. et al. Efeito das diferentes fases do ciclo menstrual no desempenho da força muscular em 10RM. Revista Brasileira de Medicina do Esporte, v. 17, n. 1, p. 22-25, 2011.

MACKENZIE, B. 101 Performance evaluation test. London: Electric word, 2005. 229p.

MATERKO, W.; NEVES, C. E. B.; SANTOS, E. L. Modelo de predição de uma repetição máxima (1RM) baseado nas características antropométricas de homens e mulheres. Revista Brasileira de Medicina do Esporte, v. 13, n. 1, p. 27-32, 2007.

MELEGÁRIO, S. M. et al. A influência do ciclo menstrual na flexibilidade em praticantes de ginástica de academia. Revista Brasileira de Medicina do Esporte, v. 12, n. 3, p. 125-128, 2006.

PALLAVI, L. C.; SOUZA, U. J. D.; SHIVAPRAKASH, G. Assessment of Musculoskeletal Strength and Levels of Fatigue during Different Phases of Menstrual Cycle in Young Adults. Journal of clinical and diagnostic research: JCDR, v. 11, n. 2, p. CC11, 2017.

SAKAMAKI, M.; YASUDA, T.; ABE, T. Comparison of low-intensity blood flow-restricted training-induced muscular hypertrophy in eumenorrheic women in the follicular phase and luteal phase and age-matched men. Clin Physiol Funct Imaging, v. 32, n. 3, p. 185-191, 2012.

SAKAMAKI-SUNAGA, M. et al. Effects of Menstrual Phase - Dependent Resistance Training Frequency on Muscular Hypertrophy and Strength. The Journal of Strength \& Conditioning Research, v. 30, n. 6, p. 1727-1734, 2016.

SARWAR, R.; NICLOS, B. B.; RUTHERFORD, O. M. Changes in muscle strength, relaxation rate and fatiguability during the human menstrual cycle. The Journal of physiology, $v$. 493, n. 1, p. 267-272, 1996.

SCHAUMBERG, Mia A. et al. Oral Contraceptive Use Dampens Physiological Adaptations to Sprint Interval Training. Medicine and science in sports and exercise, v. 49, n. 4, p. 717 727, 2017.

SCHELLENBERG, R. Treatment for the premenstrual syndrome with agnus castus fruit extract: prospective, randomised, placebo controlled study. Bmj, v. 322, n. 7279, p. 134-137, 2001. 
SIMÃO, R. et al. Variações na força muscular de membros superior e inferior nas diferentes fases do ciclo menstrual. Revista Brasileira de Ciência e Movimento, v. 15, n. 3, p. 47-52, 2007.

THOMAS, J. R.; NELSON, J. K.; SILVERMAN, S. J. Métodos de pesquisa em atividade física. Porto Alegre: Artmed Editora, 2002.

TIMONEN, S.; PROCOPÉ, B.-J. Premenstrual syndrome and physical exercise. Acta obstetricia et gynecologica Scandinavica, v. 50, n. 4, p. 331-337, 1971.

VANDER, A.; SHERMAN, J.; LUCIANO, D. Human physiology. The Mechanisms of body function. $8^{\text {th }}$ ed. New York: Mc Graw-Hill, 2001.

VISHNUPRIYA, R.; RAJARAJESWARAM, P. Effects of aerobic exercise at different intensities in pre menstrual syndrome. The Journal of Obstetrics and Gynecology of India, v. 61, n. 6, p. 675-682, 2011.

WIKSTRÖM-FRISÉN, L.; BORAXBEKK, C.-J.; HENRIKSSON-LARSÉN, K. Effects on power, strength and lean body mass of menstrual/oral contraceptive cycle based resistance training. Journal of Sports Medicine and Physical Fitness, v. 57, p. 43-52, 2017a.

WIKSTRÖM-FRISÉN, L.; BORAXBEKK, C.-J.; HENRIKSSON-LARSÉN, K. Increasing training load without risking the female athlete triad: menstrual cycle based periodized training may be an answer? Journal of Sports Medicine and Physical Fitness, v. 57, p. 1519$1525,2017 b$.

WOJTYS, E. M. et al. Association between the menstrual cycle and anterior cruciate ligament injuries in female athletes. The American journal of sports medicine, v. 26, n. 5, p. 614619, 1998.

Recebido em: 26/10/2017

Revisado em: 23/12/2017

Aprovado em: 02/01/2018

Endereço para correspondência:

vcoswig@gmail.com

Victor Silveira Coswig

Universidade Federal do Pará, Campus I Castanhal - Faculdade de Educação Física

Av. dos Universitários, $\mathrm{s} / \mathrm{n}$

Jaderlândia, Castanhal - PA, 68746-630 